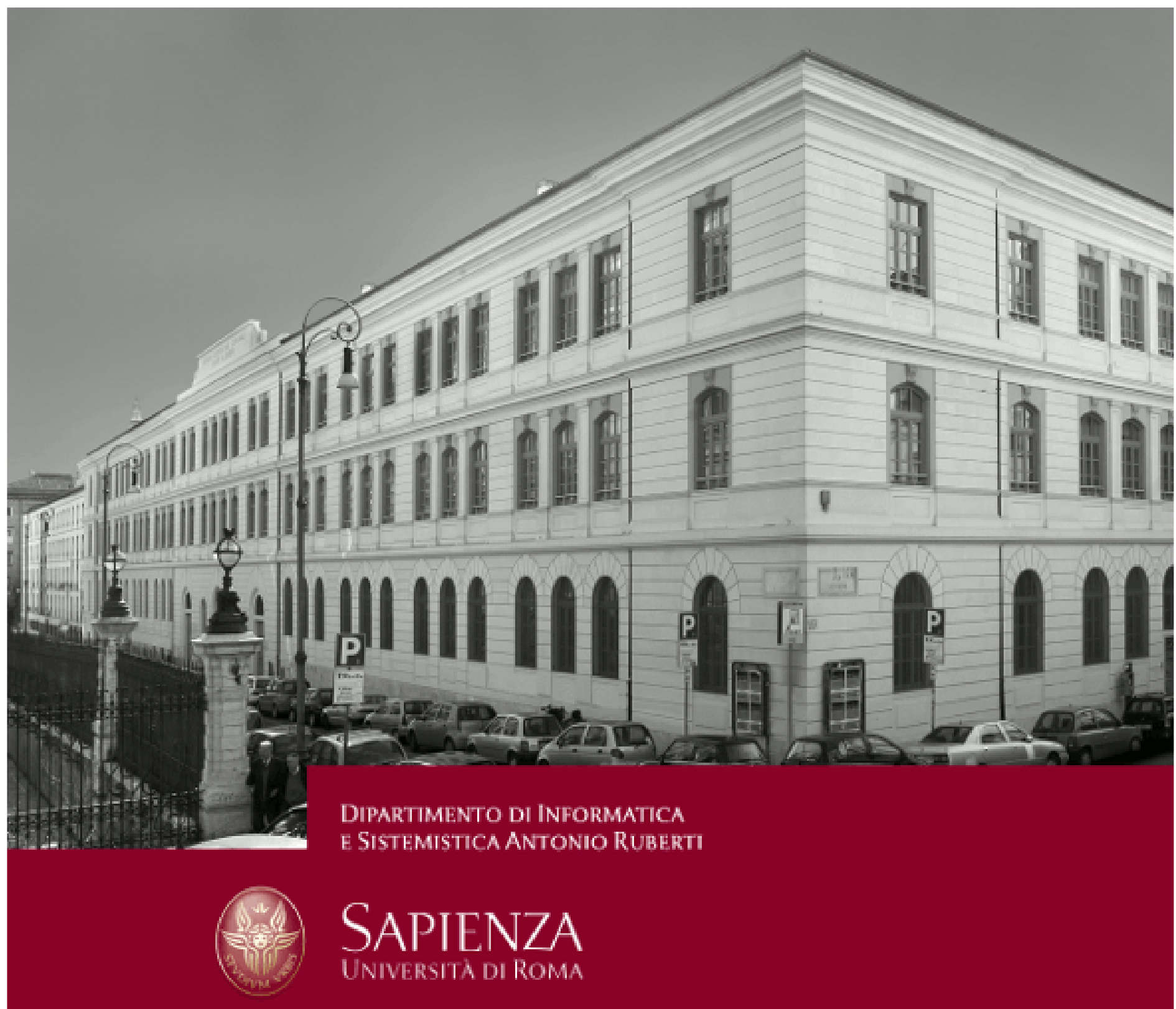

Planning Wireless Networks by Shortest Path

Carlo Mannino

Sara Mattia

Antonio Sassano

Technical Report n. 2, 2009 


\title{
Planning Wireless Networks by Shortest Path *
}

\author{
C. Mannino, S. Mattia, A. Sassano ${ }^{\dagger}$
}

\begin{abstract}
Transmitters and receivers are the basic elements of wireless networks and are characterized by a number of radio-electrical parameters. The generic planning problem consists in establishing suitable values for these parameters so as to optimize some network performance indicator. The version here addressed, namely the Power Assignment Problem (PAP), is the problem of assigning transmission powers to the transmitters of a wireless network so as to maximize the satisfied demand. This problem has relevant practical applications both in radio-broadcasting and in mobile telephony. Typical solution approaches make use of mixed integer linear programs with huge coefficients in the constraint matrix yielding numerical inaccuracy and poor bounds and cannot be exploited to solve large instances of practical interest. In order to overcome these inconveniences, we developed a two-phase heuristic to solve large instances of PAP , namely a constructive heuristic followed by an improving local search. Both phases are based on successive shortest path computations on suitable directed graphs. Computational tests on a number of instances arising in the design of the Italian Digital Video Broadcasting are presented.
\end{abstract}

Keywords: wireless network optimization, mixed integer programs, exponential neighborhood search.

*Partially supported by MIUR, Project 2006015972, Optimization models and algorithms for the design of wireless networks.

${ }^{\dagger}$ Dipartimento di Informatica e Sistemistica, Università di Roma "Sapienza", e-mail: mannino[mattia, sassano]@dis.uniroma1.it 


\section{Introduction}

A wireless network consists of a set of radio transmitters distributing services to a set of receivers scattered over a target area. Transmitters and receivers are characterized by their geographical position and by a number of radio-electrical parameters. Due to the very large number of receivers and to the uncertainty on their exact location, several neighboring receivers are typically grouped into a single representative one. A standard aggregation technique consists in subdividing the area of interest (target area) into a set of smaller rectangular areas, called testpoints. Each testpoint resumes the behavior of all receivers in the square.

As recommended by international standardization bodies, the testpoints "grid" is universally adopted as a model to evaluate the quality of the service offered by wireless networks, both in the planning and in the operational phase. In particular, the service offered by the transmitters of the network is evaluated in the center of each testpoint; each testpoint is assigned to a specific transmitter and is covered if (a measure of) the quality of the received (useful) signal exceeds a prescribed threshold value. Due to this practice, most optimization models also refer (often implicitly) to the testpoints grid $([4,10])$. The optimization process consists in establishing suitable values for a subset of the radio-electrical parameters associated to the transmitters and the receivers of the network. The remaining parameters are fixed to some reference value. Different (versions of) wireless network planning problems stem out from different parameters configurations [10]. In most cases the objective is to maximize the number (or the population) of the covered testpoints. Several algorithms have been developed to solve different planning problems. Many of these are based on Mixed Integer Linear Programming (MILP) formulations (see [3, 8, 9, 11] for mobile planning, [10, 14] for broadcasting). However, as pointed out by several authors (see, for example, [11]), these formulations suffer from weakness of the respective bounds and, even worst, from numerical inaccuracy: in practice, they fail to solve even small/medium sized instances of practical interest.

The problem here addressed, namely the Power Assignment Problem (PAP), is the problem of establishing transmission powers and testpoint assignments to the active transmitters so as to maximize the covered population, when all other radio-electrical parameters (of transmitters and receivers) are fixed. Natural instances of the PAP arise in the standard planning process of large broadcasting networks. In particular, when re-planning of operating networks must be undertaken in order to satisfy new constraints imposed by network adjustments, new international agreements, or by the introduction of new devices. Indeed, the application which motivated this research is the actual replacement, in broadcasting networks, of the analog technology with the digital one, which is occurring in Italy and all over Europe. The existence of already operating networks and the restrictions imposed by a recent international agreement ([13]) strongly constraint the planning problem, which can be reduced to solving a sequence of independent PAPs, namely one for each available transmission frequency. The corresponding instances are still too large to be attacked by exact methods, and we resorted to heuristic approaches. In particular, we show that, under specific conditions, PAP can be reduced to identify and delete the negative weight directed cycles of a suitable graph. By relaxing the original problem these conditions can be met and the corresponding graph is built. Both negative cycle detection and final power assignment are found by means of a standard shortest path algorithm. To improve the quality of this initial solution, we developed a local search approach by defining an effective exponential neighborhood ([1]) which may 
be searched in poly-time, again by solving a sequence of shortest path problems on suitable directed graphs.

The paper is organized as follows. The basic technological elements are introduced in Section 2 along with the formal statement of the PAP. In Section 3 we show how to heuristically solve PAP by shortest path computations. In Section 4 we describe the exponential neighborhood search. Finally, in Section 5 we give computational results over a number of real-life instances arising in the re-planning of the Italian national broadcasting network.

\section{The Power Assignment Problem}

A wireless network distributes its services from a set $T$ of transmitters to receivers over a portion of territory, referred to as target area. The transmitter configuration is defined by a number of physical and radio-electrical parameters, such as geographical location, activation state (on/off), transmission frequency, emission power, polarization, antenna tilt, time delay, etc. Receivers need also to be configured; most often, the only parameter taken into account is the antenna orientation, which in turn depends on the choice of a reference transmitter (server). The great majority of the approaches presented in the literature consider emission powers and/or frequencies and/or server assignments as main decision variables, while all other parameters are fixed to some pre-defined values or neglected. In this context, since transmission frequencies get fixed to reference values, the decision variables are associated with emission powers and reference transmitters.

The power emitted by a transmitter in every direction is described by its antenna diagram or radiation pattern. The radiation pattern is the two- or three-dimensional spatial distribution of radiated energy as a function of the observer's position along a path or surface of constant radius $([5])$.

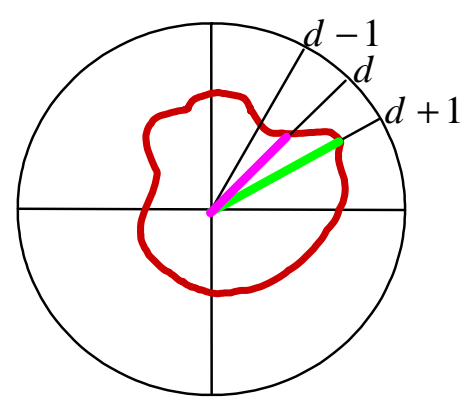

Figure 1: Antenna Diagram: example

Usually, for network planning purposes only horizontal diagrams are taken into account. The angular dependence of the horizontal radiation patterns is approximated by specifying thirty-six values attached to angles from 10 degrees to $360^{\circ}([6])$ : the corresponding directions are numbered from 1 to 36 . Consequently, the radiation pattern of each transmitter can be described by a set of 36 variables, each representing the power emitted in a specific direction. So, for all $t \in T$, let $D_{t}=\{(t, d): d=1, \ldots, 36\}$ be the set of directions of $t$ : we introduce a power variable $p_{s}$ for all $s \in D_{t}$, each ranging in the interval $\left[\epsilon, P_{\text {Max }}\right]$, where $\epsilon>0$ is a positive small constant. We assume that if $p_{s}=\epsilon$ for all $s \in D_{t}$, then $t$ is switched off. Each 
element $(t, d) \in D_{t}$ can be regarded as an "elementary transmitter", later referred to as a $d$-transmitter. In order to yield feasible antenna diagrams, the powers $p_{s}: s \in D_{t}$ emitted by each $d$-transmitter of a same transmitter $t$ must obey simple technological laws ([6]): namely, their ratio cannot exceed specified thresholds, which can be expressed by following family of inequalities, denoted as design constraints:

$$
\frac{p_{s}}{p_{q}} \leq \Delta_{s q} \quad t \in T, \quad s, q \in D_{t}
$$

In particular, we consider two types of design constraints. Those involving only adjacent directions $s=(t, i), q=(t, i+1)$, for $i=1, \ldots, 36$, with $\Delta_{s q}=\Delta_{a d j}=10^{0.5}$ and those between any pair of directions $s, q \in D_{t}$, for which $\Delta_{s q}=\Delta=10^{2.4}$. Lower and upper bounds are represented by the following family of linear constraints:

$$
\epsilon \leq p_{s} \leq P_{\text {Max }} \quad t \in T, \quad s \in D_{t}
$$

A power vector satisfying all design constraints (1) and all bounds (2) is said to be feasible.

In order to evaluate the quality of the received signals, the target area is decomposed into a set $R$ of "small", rectangular areas called testpoints (TPs). Each testpoint, identified by its coordinates, represents the behavior of all receivers within it. The number of customers in each testpoint $r \in R$ is denoted by $c_{r} \in \mathbb{Z}_{+}\left(\right.$for $\left.S \subseteq R, c(S)=\sum_{r \in S} c_{r}\right)$.

The signal emitted by a transmitter propagates according to its antenna diagram and to territory orography. The power density received in TP $r \in R$ from transmitter $t$ is proportional to the emission power $p_{s}$ of the $d$-transmitter $s=(t, d) \in D_{t}$ in the direction $d$ which is "closest" to the center of $r$. Observe that, denoting by $R(s) \subseteq R$ the set of testpoints reached by a $d$-transmitter $s$, we have $R(s) \cap R(q)=\emptyset$ for all $s, q \in D_{t}, s \neq q$, for every $t \in T$. In other words, at most one out of the $36 d$-transmitters associated with a same transmitter $t \in T$ will be received in $r \in R$. For all $r \in R$ we denote by $D(r)$ the set of $d$-transmitters received in $r$. Now, if we define $P_{r s}$ the power density received in $r \in R$ from $s \in D(r)$, we have $P_{r s}=a_{r s} \cdot p_{s}$, where coefficient $a_{r s} \in \mathbb{R}_{+}$is the fading factor and is typically calculated, for each $r \in R, s \in D(r)$, by means of a suitable propagation model (see [12]). We refer to matrix $[\mathbf{A}]=\left[a_{r s}\right]_{r \in R, s \in D(r)}$ as the fading matrix. In most cases of practical interest, the coefficients of the fading matrix may differ by several order of magnitudes (the ratio between the largest and the smallest coefficient is typically around $10^{12}$ ). For this reason, practitioners prefer to represent $a_{r s}$ by using the so called $d B$ form $a_{r s}^{d B}$, which is defined as the integer closest to the quantity $10 \log _{10} a_{r s}$. The approximation error introduced by this representation is considered tollerable for practical planning purposes. Indeed, other relevant quantities, such as emission powers and antenna design coefficients, are generally represented in $\mathrm{dB}$ form. In particular, we have $\Delta_{a d j}^{d B}=5 d B$ and $\Delta^{d B}=24 d B$.

We briefly describe now how service quality assessment is carried out. Informally, a TP $r$ is said to be covered if the service is received in $r$ above a given quality level. Among all $d$ transmitters received in a TP, exactly one is selected as the reference transmitter (or reference signal or simply server), the major (possibly the unique) candidate to ensure the service in the 
TP. Then, the coverage condition is expressed by the following inequality, denoted as Signal to Interference Ratio inequality:

$$
\frac{\sum_{s \in U\left(r, h_{r}\right)} P_{r s}}{\sum_{s \in I\left(r, h_{r}\right)} P_{r s}} \geq b_{r},
$$

where constant $b_{r}$ is the receiver sensitivity, $h_{r}$ is the reference transmitter of $r, U\left(r, h_{r}\right) \subseteq$ $D(r)$ is the set of wanted signals in $r$ and $I\left(r, h_{r}\right) \subseteq D(r)$ is the set of interfering signals in $r$. The ratio in the left hand side of (3) is the Signal to Interference Ratio (SIR). Both sets $U\left(r, h_{r}\right)$ and $I\left(r, h_{r}\right)$ depend on the selected server $h_{r} \in D(r)$ (the exact definition of the sets $W$ and $U$ in DVB technology can be found in [10]). Once again, the threshold $b_{r}$ is provided by technical manuals in $\mathrm{dB}$ form $b_{r}^{d B}$.

Due to the relation between emitted and received powers above described, the coverage constraint for a testpoint $r$ can be rewritten as a function of the emitted powers:

$$
\frac{\sum_{s \in U\left(r, h_{r}\right)} a_{r s} p_{s}}{\sum_{s \in I\left(r, h_{r}\right)} a_{r s} p_{s}} \geq b_{r}
$$

Summarizing, for a given emission power vector $\tilde{p}$, deciding whether TP $r$ is covered or not consists in finding a reference transmitter $h_{r} \in D(r)$ satisfying (4) or proving that none exists. Therefore, coverage evaluation in TP $r$ is carried out by enumerating all $|D(r)|$ SIRs inequalities associated with the candidate reference transmitters. If $r$ is covered, the receiver chooses as reference transmitter the one maximizing the SIR. The proof of the following remark can be found in [10]:

Remark 2.1 [10] The coverage evaluation procedure can be carried out in linear (amortized) time $O(|D(r)|)$.

We are now able to introduce a more formal statement of our specific planning problem. Let $D=\{(t, d): t \in T, d=1, \ldots, 36\}$. An assignment $h \in D^{|R|}$ of reference transmitters to all TPs is called server assignment.

Problem 2.2 (Power Assignment Problem (PAP)) Given a network $(T, R)$, the fading matrix $[A]_{s \in D(r), r \in R}$ and a power upper bound $P_{\text {Max }} \in \mathbb{R}_{+}$; suppose that a transmission frequency is given for each $t \in T$. Find a server assignment $h \in D^{|R|}$ and a feasible power vector $p \in \mathbb{R}^{|D|}$ such that the population $c(p, h)$ of the covered testpoints is maximized.

\section{Reducing Power Assignment to shortest path}

Problem 2.2 can be readily cast into a Mixed Integer Linear Program (see, for example, [10]). However, it is common experience that the MILP formulations corresponding to instances of some practical interest are far from being solvable by standard Branch\&Cut, for a number of reasons. First, these instances contain a large number of binary variables, which results in huge search trees. Second, the coefficient matrix is ill conditioned as the entries may differ by several orders of magnitudes. As a consequence, the time to perform standard simplex operations increases and, even worst, the solutions produced by the lp solver are not always 
reliable. Third, the constraints associated with the SIR inequalities contain the notorious $B I G_{-} M$ coefficient which may yield poor relaxations. For this reason we decided to resort to an effective heuristic approach. In order to introduce our methodology we need the following:

Definition 3.1 A set of testpoints $S \subseteq R$ is coverable iff there exist a server assignment $h$ and a feasible power vector $p$ such that all testpoints in $S$ are covered.

The above definition reduces for PAP to finding $\bar{h} \in D^{R}$ and $\bar{p} \in \mathbb{R}^{D}$ satisfying the following system of inequalities:

$$
\begin{array}{ll}
\operatorname{COV}(S, \bar{h}) & \\
\frac{\sum_{s \in U\left(r, \bar{h}_{r}\right)} a_{r s} \bar{p}_{s}}{\sum_{s \in I\left(r, \bar{h}_{r}\right)} a_{r s} \bar{p}_{s}} \geq b_{r} & r \in S \\
\frac{\bar{p}_{s}}{\bar{p}_{q}} \leq \Delta_{s q} & t \in T, s, q \in D_{t} \\
\epsilon \leq \bar{p}_{s} \leq P_{\text {Max }} & t \in T, s \in D_{t}
\end{array}
$$

We can now rephrase PAP as the problem of finding a set $S \subseteq R$ of testpoints and a server assignment $h \in D^{R}$ such that $\operatorname{COV}(S, h)$ is feasible and the population $c(S)$ of $S$ is maximum.

Let us consider now the following family of linear inequalities:

$$
C O V(R)
$$

$$
\begin{array}{ll}
\frac{\sum_{s \in U\left(r, h_{r}\right)} a_{r s} p_{s}}{\sum_{s \in I\left(r, h_{r}\right)} a_{r s} p_{s}} \geq b_{r} & r \in R, h_{r} \in D(r) \\
\frac{p_{s}}{p_{q}} \leq \Delta_{s q} & t \in T, s, q \in D_{t} \\
\epsilon \leq p_{s} \leq P_{\text {Max }} & t \in T, s \in D_{t}
\end{array}
$$

It is easy to see that, for all possible $S \subseteq R$ and all server assignment $h$, with $h_{r} \in D(r)$, we have $\operatorname{COV}(S, h) \subseteq C O V(R)$. Thus PAP can be rephrased as the problem of finding a suitable subset of constraints (8) to remove so that the remaining family of constraints:

(i) is feasible;

(ii) contains at most one SIR inequality for each $r \in R$

and the (iii) the population of the associated testpoints is maximum.

Our heuristic approach to the solution of PAP is based on a number of simplifying but quite reasonable technological assumptions. First observe that, due to the wide variability of the fading coefficients and emission powers, the signals received in a testpoint typically differ one from another by order of magnitudes. More specifically, for each $r \in R$, if $r$ is 
covered with reference signal $h_{r} \in D(r)$, in most cases there will be only one "strongest" useful signal, namely $h_{r}$, and the contribution of the other useful signals to the numerator of the SIR inequality (4) can be neglected. So, if we assume that this condition is always satisfied, we rewrite (8) as:

$$
\frac{a_{r h_{r}} p_{h_{r}}}{\sum_{s \in I\left(r, h_{r}\right)} a_{r s} p_{s}} \geq b_{r} \quad r \in R, h_{r} \in D(r)
$$

and $r$ is covered if at least one of the $(|D(r)|)$ constraints (11) associated with its potential servers is satisfied. Similarly, if we assume that for each $r \in R, h_{r} \in D(r)$ there will be only one strongest interfering signal, all other interfering signals can be neglected. However, since emission powers are not known in advance, for each $r \in R, h_{r} \in D(r)$, we split (11) into $\left|I\left(r, h_{r}\right)\right|$ inequalities of the form

$$
\frac{a_{r h_{r}} p_{h_{r}}}{a_{r t} p_{t}} \geq b_{r} \quad t \in I\left(r, h_{r}\right)
$$

and $r$ is covered with reference signal $h_{r}$ iff all of the constraints (12) associated with $r$ and $h_{r}$ are satisfied.

Now, similarly to $\mathrm{COV}(R)$, if we define $\mathrm{COV}_{2}(R)$ as the following family of constraints:

$$
\begin{aligned}
& \mathrm{COV}_{2}(\mathrm{R}) \\
& \frac{a_{r h_{r}} p_{h_{r}}}{a_{r t} p_{t}} \geq b_{r} \quad r \in R, h_{r} \in D(r), t \in I\left(r, h_{r}\right) \\
& \frac{p_{s}}{p_{q}} \leq \Delta_{s q} \quad t \in T, s, q \in D_{t} \\
& \epsilon \leq p_{s} \leq P_{\text {Max }} \quad t \in T, s \in D_{t}
\end{aligned}
$$

then PAP can be rephrased as the problem of removing a suitable subset of constraints of type (13) from $\mathrm{COV}_{2}(\mathrm{R})$ so that the remaining constraints:

(i) are feasible;

(ii) contain at most one family of SIR inequalities for each $r \in R$, namely the family associated with a specific reference signal $h_{r} \in D(r)$, and all the associated interferers $t \in I\left(r, h_{r}\right)$.

and, in addition, (iii) the population of the covered testpoints is maximized.

Observe now that $\mathrm{COV}_{2}(R)$ can be rewritten in the following equivalent way:

$$
C O V_{2}^{d B}(R)
$$

$$
\begin{array}{rl}
10 \log _{10} \frac{a_{r h_{r}} p_{h_{r}}}{a_{r t} p_{t}} \geq 10 \log _{10} b_{r} & r \in R, h_{r} \in D(r), t \in I\left(r, h_{r}\right) \\
10 \log _{10} \frac{p_{s}}{p_{q}} \leq 10 \log _{10} \Delta_{s q} & t \in T, s, q \in D_{t} \\
10 \log _{10} \epsilon \leq 10 \log _{10} p_{s} \leq 10 \log _{10} P_{M a x} & t \in T, s \in D_{t}
\end{array}
$$


By introducing, for all $t \in T, s \in D_{t}$, a variable $p_{s}^{d B}=10 \log _{10} p_{s}$, and by expressing all constants in $\mathrm{dB}$ form, by simple algebra we obtain:

$$
\begin{aligned}
& C O V_{2}^{d B}(R) \\
& p_{h_{r}}^{d B}-p_{t}^{d B} \geq b_{r}^{d B}+a_{r h_{r}}^{d B}-a_{r t}^{d B} \quad r \in R, h_{r} \in D(r), t \in I\left(r, h_{r}\right) \\
& p_{s}^{d B}-p_{q}^{d B} \leq \Delta_{s q}^{d B} \quad t \in T, s, q \in D_{t} \\
& \epsilon^{d B} \leq p_{s}^{d B} \leq P_{\text {Max }}^{d B} \quad t \in T, s \in D_{t}
\end{aligned}
$$

By adding an extra, reference power variable $p_{0}$, we can replace each (21) with a pair

$$
\begin{aligned}
& p_{s}^{d B}-p_{0} \leq P_{\text {Max }}^{d B} \\
& p_{0}-p_{s}^{d B} \leq-\epsilon^{d B}
\end{aligned}
$$

Thus, $C_{O} V_{2}^{d B}(R)$ can be rewritten in compact form as

$$
p_{j}^{d B}-p_{i}^{d B} \leq l_{i j} \quad i j \in A .
$$

The family of solutions to (24) is the solution set of the dual of a shortest path problem on the weighted graph $G^{d B}(D, R)=(V, A, l)$, with $V=D \cup\{0\}$. Each arc $i j \in A$ corresponds to one of the constraints of $C O V_{2}^{d B}$ : a testpoint arc is an arc corresponding to a constraint of type (19). If $\left(r, h_{r}, t\right)$ is a constraint of type (19), we denote by $a\left(r, h_{r}, t\right)$ the corresponding testpoint arc. It is well known (see, for example, [2]) that $(24)$ has a solution iff $G^{d B}(D, R)$ does not contain a negative weight directed cycle. Also observe that each negative cycle in $G^{d B}(D, R)$ corresponds to a infeasible subsystem of $C O V_{2}^{d B}(R)$. We have the following:

Lemma 3.2 Let $C$ be a negative cycle of $G(V, A, l)$. Then $C$ contains a testpoint arc.

Proof. Let $\tilde{p}_{s}^{d B}=\epsilon^{d B}$ for all $s \in D$, and let $\tilde{p}_{0}=0$. It is easy to see that $\tilde{p}$ satisfies constraints (20), (22) and (23). As a consequence, any infeasible subsystem of $C O V_{2}^{d B}$ contains at least one constraint of type (19).

The above lemma is the basis of a heuristic procedure to find a feasible subsets of constraints of $C O V_{2}^{d B}$. The idea is to iteratively identify a negative weight dicycle in $G^{d B}(D, R)$ and remove suitable subset of testpoint arcs meeting the negative dicycles. In particular, if $C$ is a negative dicycle, then we select a testpoint arc $a\left(r, h_{r}, t\right) \in C$ and remove it from the graph. Intuitively, this corresponds to renounce covering $r$ with reference signal $h_{r}$. As a consequence, all testpoint arcs corresponding to the different interferers $I\left(r, h_{r}\right)$ of $r$ and $h_{r}$ must also be dropped from $G^{d B}(D, R)$. The procedure stops when no negative dicycles are left. The following scheme summarizes what above discussed:

Procedure Cycle_Detect

1. Build the graph $G^{0}=G(R, D)=(V, A, l)$. 
2. While $G^{i}$ contains a negative weight dicycle $C^{i}$.

(a) Choose a testpoint arc $a\left(r, h_{r}, t\right)$ in $C^{i}$.

(b) Build $G^{i+1}$ by deleting all testpoint arcs corresponding to $r, h_{r}$, i.e. remove the arc set $A\left(r, h_{r}\right)=\left\{a\left(r, h_{r}, t\right) \in G^{i}: t \in I\left(r, h_{r}\right)\right\}$.

(c) $\mathrm{i}:=\mathrm{i}+1$;

3. EndWhile.

4. Let $q=i$. Compute the shortest path lengths $\tilde{p}_{s}$ from 0 to $s \in V$ in $G^{q}$.

Observe that Step 2.a and Step 2.b correspond to identifying a testpoint $r$, with reference signal $h_{r}$ and remove the corresponding constraints from $C O V_{2}^{d B}$; recall that there are $\left|I\left(r, h_{r}\right)\right|$ such constraints. Let $G^{q}\left(V, A^{q}\right)$ be the final graph, and let $A_{R}^{q}$ be its testpoint arcs. Denote by $R\left(G^{q}\right)$ the family of testpoints corresponding to some arcs in $G^{q}$, i.e. $R\left(G^{q}\right)=\left\{r \in R: \exists a\left(r, h_{r}, t\right) \in A^{q}\right\}$. Finally denote by $C O V_{2}^{d B}\left(G^{q}\right)$ the family of constraints corresponding to the $\operatorname{arcs}$ of $G^{q}$. Observe that the solution $\tilde{p}$ returned by Procedure Cycle_Detect is feasible for $\mathrm{COV}_{2}^{d B}\left(G^{q}\right)$.

Lemma 3.3 Let $\tilde{p}^{d B}$ be a feasible solution to $C O V_{2}^{d B}\left(G^{q}\right)$. Then all testpoints in $R\left(G^{q}\right)$ are covered.

Proof. By construction, if $a\left(\bar{r}, \bar{h}_{r}, \bar{t}\right) \in A^{q}$, then $a\left(\bar{r}, \bar{h}_{r}, t\right) \in A^{q}$ for all $t \in I\left(\bar{r}, \bar{h}_{r}\right)$. This implies that $\mathrm{COV}_{2}^{d b}\left(G^{q}\right)$ contains all constraints (19) associated with $\bar{r}, \bar{h}_{r}$, and $\bar{r}$ is covered with reference signal $\bar{h}_{r}$.

The identification of a negative dicycle $C^{i}$ in $G^{i}$ can be performed by applying the BellmanFord algorithm, which either finds $C^{i}$ or returns a feasible solution $\tilde{p}$ to $C O V_{2}^{d b}\left(G^{i}\right)$. In order to establish how to select the arc at Step 2a. we tested several criteria. The best one corresponds to selecting the arc which appeared most often in the negative cycles detected so far; ties are broken by selecting the one minimizing the population of the corresponding testpoint.

\section{Exponential Neighborhood Search}

The solution $(\tilde{p}, \tilde{h})$ to PAP returned by Procedure Cycle_Detect is computed by neglecting the effect of multiple useful and interfering transmitters. In order to increase its quality we developed an efficient exponential neighborhood search which takes into account these contributions to the actual SIR.

Neighborhood structure Let $(\bar{p}, \bar{h})$ be the current solution. For any $\bar{t} \in T$ define $N_{\bar{t}}(\bar{p}, \bar{h})=$ $\{(\tilde{p}, \tilde{h})\}$ as the family of solutions obtained by letting $\tilde{p}_{s}=\bar{p}_{s}$ for every $s \in D_{q}, q \in T-\{\bar{t}\}$. In other words, $N_{\bar{t}}(\bar{p}, \bar{h})$ is the family of solutions which can be obtained from the original one by changing the power vector $p_{\bar{t}}=\left(p_{1, \bar{t}}, \ldots, p_{36, \bar{t}}\right)$ associated with the directions $d \in D_{t}$ of a single transmitter $\bar{t} \in T$, and re-assigning reference signals in all possible ways. We suppose that, for all $t \in T$ and $s \in D_{t}, p_{s}^{d B}$ belongs to a discrete set of integer $d B$ values $\mathcal{L}=\left\{L_{1}, \ldots, L_{q}\right\}$. Finally, we define the neighborhood $N(\bar{p}, \bar{h})$ of a solution $(\bar{p}, \bar{h})$ as $N(\bar{p}, \bar{h})=\bigcup_{t \in T} N_{t}(\bar{p}, \bar{h})$. 
Neighborhood search Exploring the neighborhood $N(\bar{p}, \bar{h})$ consists in searching each $N_{t}$, for all $t \in T$, and then choosing the best configuration encountered. Searching $N_{t}$ is equivalent to finding the configuration $\left(p^{*}, h^{*}\right)_{t} \in N_{t}(\bar{p}, \bar{h})$ so that $c\left(\left(p^{*}, h^{*}\right)_{t}\right)$ is maximized. Since powers are fixed for all $z \in T-\{t\}$, we only need to establish the best power vector $p_{t}^{*}=\left(p_{t, 1}, \ldots, p_{t, 36}\right)^{*}$ for $t$ and the corresponding new reference signals $h^{*} \in D^{R}$. Specifically, $p_{t}^{*}$ must be feasible - i.e. satisfy all adjacent and non-adjacent design constraints (1) - and must maximize coverage.

Observe that the number of different feasible vectors $h \in N_{t}$ grows exponential in $|R|$ and $|T|$, being in correspondence with the feasible assignments of TPs to reference signals. However, we will show that the optimum solution in $N_{t}$ can be found in polynomial time (in $|R|$ and $|\mathcal{L}|)$. Let $c_{\epsilon}^{t}(\bar{p}, \bar{h})$ be the coverage of the solution obtained by $(\bar{p}, \bar{h})$ by switching off $t \in T$, i.e. by letting $\left(p_{t, 1}=\ldots=p_{t, 36}=\epsilon\right)$. Denote by $c_{d k}$ the coverage increase in direction $d$ with respect to $c_{\epsilon}^{t}(\bar{p}, \bar{h})$ when $p_{t, d}^{d B}=L_{k} \in \mathcal{L}$. This coefficient can be efficiently computed by the coverage evaluation procedure described in Section 2 (Remark 2.1) and it can assume positive, zero or negative value. Recall that the coverage evaluation procedure also establishes, for each testpoint $r \in R(t, d)$ (the family of testpoints reached by the $d$-direction of $t$ ), the corresponding reference signal $\tilde{h}_{r}$. Finally, recall that $R\left(t, d_{1}\right) \cap R\left(t, d_{2}\right)=\emptyset$ whenever $d_{1} \neq d_{2}$.

Now, in order to find the optimum solution in $N_{t}(\bar{p}, \bar{h})$, we first find the optimum solution when the power of $t$ in its first direction $(t, 1)$ is fixed to some reference value (in $\mathrm{L}$ ). In other words, We want to find the optimum configuration for $t$ in $N_{t}(\bar{p}, \bar{h})$ when $p_{(t, 1)}^{d B}=L_{k} \in \mathcal{L}$. We show that this can be done by solving a sequence of shortest path problems in a suitable acyclic directed graph $G_{k}=G\left(k, \Delta_{a d j}\right)=\left(V_{k}, A_{k}\right)$, for $k=1, \ldots, q$. Each vertex $v_{d, \ell} \in V_{k}$ of $G_{k}$ is associated with a direction $d$ and a feasible power level $L_{\ell} \in \mathcal{L}$. In particular, $v_{1, k} \in V_{k}$, i.e. there is a vertex associated with direction 1 and power level $L_{k}$; then we have a vertex for every other direction and every power level in $\mathcal{L}$, namely $v_{d \ell} \in V_{k}, 2 \leq d \leq 36, \ell=1, \ldots, q$. Finally, $V_{k}$ contains an extra node $w$. The arcs of $G_{k}$ are associated with the pair of power levels satisfying the adjacency design constraints $p_{s}^{d B}-p_{u}^{d B} \leq \Delta_{a d j}^{d B}$ for all adjacent directions $s$ and $u$. Namely, for $d=1, \ldots, 35,\left(v_{d, \ell}, v_{d+1, g}\right) \in A_{k}$ iff $\left|L_{\ell}-L_{g}\right| \leq \Delta_{a d j}^{d B} ;\left(v_{36, \ell}, w\right) \in A_{k}$ for all $v_{36, \ell} \in V_{k}$ such that $\left|L_{\ell}-L_{k}\right| \leq \Delta_{a d j}^{d B}$. Finally, with every arc $\left(v_{d, \ell}, v_{d+1, g}\right)$ we associate the weight $c_{d \ell}$.

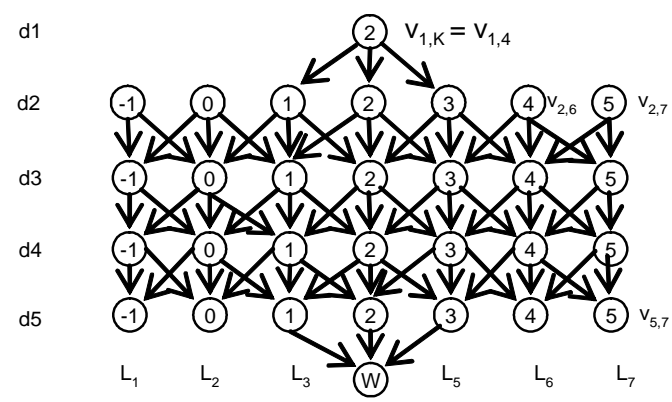

Figure 2: Example of neighborhood graph

An example of this construction is shown in Fig.2, where, for the sake of simplicity, we have supposed only 5 directions, 7 power levels $\mathcal{L}=\left\{L_{1}=-1, L_{2}=0, L_{3}=1, L_{4}=2, L_{5}=\right.$ $\left.3, L_{6}=4, L_{7}=5\right\}$, and $\Delta_{a d j}^{d B}=1$. 
It is easy to see that $G_{k}$ is a layered graph. Also, it is immediate to verify that any power vector $\left(p_{t, 1}^{d B}, \ldots, p_{t, 36}^{d B}\right) \in \mathcal{L}^{36}$ satisfying $p_{t, 1}^{d B}=L_{k}$ and all the adjacency design constraints, corresponds to the directed path $m=\left\{v_{1, k}, v_{2, p_{t, 2}}, \ldots, v_{36, p_{t, 36}}, w\right\}$. Moreover, the weight of $m$ is precisely the coverage increase with respect to $c_{\epsilon}^{t}(\bar{p}, \bar{h})$ when $t$ is assigned powers $p_{t, 1}^{d B}, \ldots, p_{t, 36}^{d B}$. Analogously, it is easy to see that any directed path $\tilde{m}=\left(v_{1, k}, v_{2, \ell_{2}}, \ldots, v_{36, \ell_{36}}, w\right)$ in $G_{k}$ corresponds to a power assignment for $t$, namely $\tilde{p}_{t, 1}^{d B}=L_{k}, \ldots, \tilde{p}_{t, 36}^{d B}=L_{\ell_{36}}$, satisfying all adjacency design constraints (1). Recall now that feasible power assignments must satisfy both adjacency and non-adjacency design constraints. We have the following:

Lemma 4.1 Suppose $\tilde{m}$ is a maximum weighted path in $G_{k}$, and suppose the corresponding power assignment $\tilde{p}^{t}=\left(\tilde{p}_{t, 1}^{d B}, \ldots \tilde{p}_{t, 36}^{d B}\right)^{\mathrm{T}}$ also satisfies all non-adjacent design constraints, then $\tilde{p}^{t}$ is optimum in $N_{t}(\bar{p}, \bar{h})$.

As a consequence, a maximum path $m_{k}^{*}$ in $G_{k}$ whose corresponding power assignment $p_{k}^{*}$ satisfies the non-adjacent design constraints solves the optimization problem in $N_{t}(\bar{p}, \bar{h})$, when restricted to $p_{t, 1}^{d B}=L_{k}$. Since $G_{k}$ is layered, $G_{k}$ is acyclic and $m_{k}^{*}$ can be computed by an $O(|A|)$ shortest path algorithm ([2]). In order to ensure that also non-adjacent design constraints are satisfied we define, for each $G_{k}, k=1, \ldots, q$ a family $G_{k}^{0}, \ldots, G_{k}^{\Delta^{d B}}$ of induced subgraphs with the property that any directed path in $G_{k}^{i}$ corresponds to a feasible power assignment and any feasible power assignment corresponds to a path in some $G_{k}^{i}$.

Namely, let $\left\{-\Delta^{d B}+L_{k}+i, \ldots, L_{k}+i\right\}$ be a set of $\Delta^{d B}$ contiguous integer power values including $L_{k}$, for $i=0, \ldots, \Delta^{d B}$. Define $\mathcal{L}^{i}=\mathcal{L} \cap\left\{-\Delta+L_{k}+i, \ldots, L_{k}+i\right\}$. By definition, there exists $i \in 0, \ldots, \Delta^{d B}$ such that every feasible power vector $\tilde{p}^{t}$, with $\tilde{p}_{t, 1}=L_{k}$ satisfies $\tilde{p}_{t, d} \in \mathcal{L}^{i}$, for all $d$. Thus, we define $G_{k}^{i}$ as the subgraph of $G_{k}$ induced by the vertices corresponding to the power levels in $\mathcal{L}_{i}$, plus vertex $w$. Any path $m$ from $v_{1, k}$ to $w$ in $G_{k}^{i}$ corresponds to a power assignment satisfying both adjacent and non-adjacent design constraints. Finally, by solving $\Delta^{d B}+1$ maximum path problems on the graphs $G_{k}^{0}, \ldots, G_{k}^{\Delta^{d B}}$ and choosing the optimum one, we find the optimum solution in $N_{t}(\bar{p}, \bar{h})$. The Neighborhood Search procedure is summarized in Table 4 .

The Neighborhood Search procedure is embedded into a standard local search (LS) approach. Namely, starting from a given initial solution, the algorithm proceeds by searching its neighborhood for an improving solution by applying Procedure Neighborhood Search: if found, this becomes the new current solution and the search continues, otherwise the search is stopped.

We apply our LS to the solution found by Procedure Cycle_Detect. However, observe that, by taking as initial solution the one with all $d$-transmitters to the minimum power $\epsilon$ (covering no testpoints), the local search can be applied to find from scratch a heuristic solution to PAP.

\section{Computational results}

We tested our heuristics on 56 real-life instances arising in the planning of the new DVB networks in north Italy. Each instance corresponds to a Single Frequency Network, operating at a specific frequency in the UHF band. These instances were generated by Fondazione Ugo Bordoni, a major Italian research foundation, advising the Italian Ministry of Communication. The instances are described in Table 2, where the name in Column name is associated 


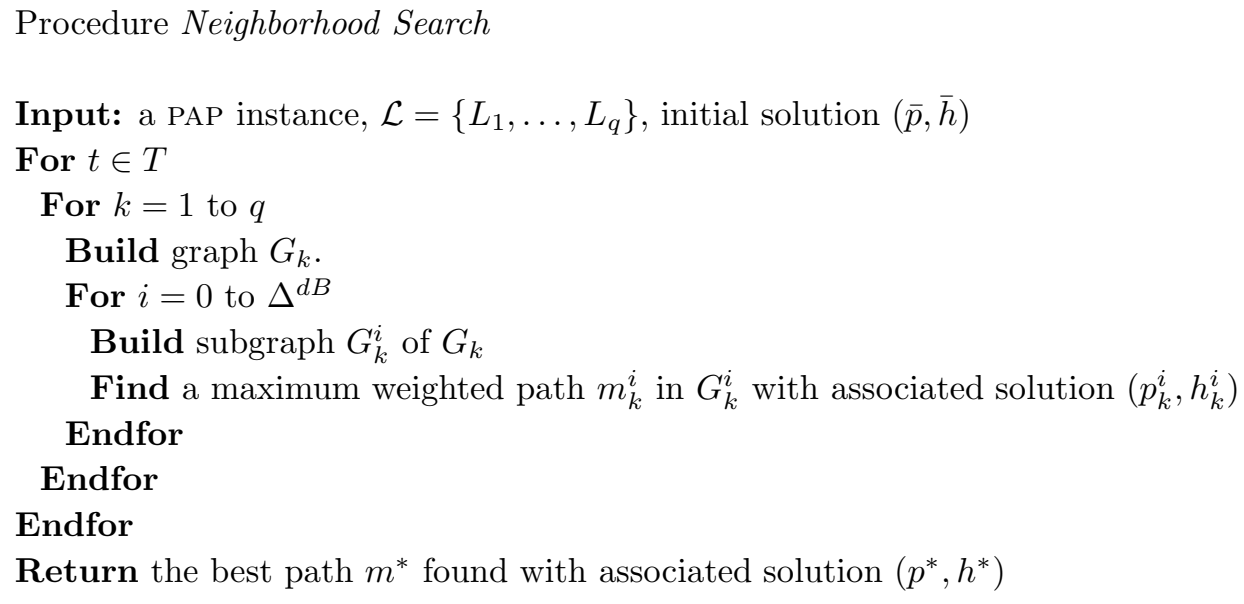

Table 1: Neighborhood Search procedure

with the transmission frequency, column population and column \# TPs is the total amount of coverable population and coverable TPs, respectively, column \# SIR is the total number of constraints (8) (corresponding to the SIR inequalities), and column \# transm. is the number of transmitters. Observe that the coverable population and TPs may differ slightly from instance to instance.

The algorithms were implemented in $\mathrm{C}++$ and run on a Intel Core 2 Duo T7500 / 2.2 $\mathrm{GHz}$, with 4 Gb RAM. We compare our heuristic, which combines the Cycle Detection (CD) procedure with the Local Search (LS) against the commercial solver ILOG-CPLEX version 11.1 ([7]), with default settings and one hour time limit, applied to a standard BIG_M formulation to PAP which slightly extends the one presented in [10] to cope with multiple candidate servers. We also compare it with the stand-alone versions of CD and LS.

The results are shown in Table 3, where column coverage is the percentage of covered population while sec. is the running time (in seconds), Column $U B$ is the Upper Bound produced by Cplex, while $L B$ is the best coverage found by Cplex.

The values show that these instances are quite difficult to solve by Branch-and-Bound, since Cplex is not able to solve any of them to optimality within time limit. For most of the instances, Cplex is not able to produce a suitable feasible solution and in 11 cases over 56, it is not even able to solve the lp relaxation. Moreover, these are regional instances, while in the future, Fondazione Ugo Bordoni intends to generate and solve larger, national instances of DVB planning.

The best approach resulted the one that combines the cycle-detection algorithm and the 
local search. In fact, it strictly dominates both CD and LS in their stand-alone versions; also, in 45 cases over 56 , it is able to produce solutions which are better than the ones found by cplex (in almost twice the running time). 


\begin{tabular}{|c|c|c|c|c|}
\hline name & population & \# TPs & \# SIR & \# transm. \\
\hline F5 & 21069661 & 10901 & 15318 & 287 \\
\hline F6 & 21369393 & 11392 & 15000 & 206 \\
\hline F7 & 21004619 & 10945 & 15241 & 250 \\
\hline F8 & 21212563 & 11284 & 15315 & 223 \\
\hline F9 & 21368196 & 11097 & 15349 & 280 \\
\hline F10 & 20922187 & 10820 & 15230 & 231 \\
\hline F11 & 21399406 & 11150 & 15304 & 246 \\
\hline $\mathrm{F} 21$ & 20530081 & 10821 & 15333 & 228 \\
\hline $\mathrm{F} 22$ & 19543530 & 10182 & 15389 & 261 \\
\hline $\mathrm{F} 23$ & 20058458 & 10371 & 15404 & 257 \\
\hline F24 & 20150831 & 10482 & 15417 & 247 \\
\hline $\mathrm{F} 25$ & 19620998 & 10034 & 15406 & 269 \\
\hline F26 & 19680288 & 10115 & 15404 & 279 \\
\hline $\mathrm{F} 27$ & 20302469 & 10762 & 15318 & 214 \\
\hline F28 & 19908252 & 10290 & 15336 & 244 \\
\hline F29 & 20268812 & 10509 & 15303 & 228 \\
\hline F30 & 19525939 & 9976 & 15419 & 279 \\
\hline F31 & 20467330 & 10774 & 15288 & 227 \\
\hline F32 & 20041155 & 10428 & 15356 & 259 \\
\hline F33 & 20138351 & 10423 & 15331 & 262 \\
\hline F34 & 19866429 & 10260 & 15370 & 254 \\
\hline F35 & 20036065 & 10383 & 15340 & 241 \\
\hline F36 & 19668580 & 10134 & 15408 & 278 \\
\hline F37 & 19903572 & 10276 & 15444 & 278 \\
\hline F38 & 19899952 & 10252 & 15436 & 288 \\
\hline F39 & 20853718 & 10812 & 15377 & 235 \\
\hline F40 & 19685609 & 10019 & 15448 & 288 \\
\hline F41 & 20244427 & 10439 & 15413 & 248 \\
\hline F42 & 20727175 & 10639 & 15396 & 261 \\
\hline F43 & 19888074 & 10229 & 15360 & 258 \\
\hline F44 & 19897282 & 10192 & 15437 & 278 \\
\hline F45 & 20257987 & 10381 & 15399 & 258 \\
\hline F46 & 20166514 & 10372 & 15390 & 257 \\
\hline $\mathrm{F} 47$ & 20751799 & 10601 & 15397 & 244 \\
\hline F48 & 20167903 & 10285 & 15471 & 278 \\
\hline F49 & 19807479 & 10079 & 15434 & 290 \\
\hline F50 & 19836959 & 10152 & 15430 & 295 \\
\hline F51 & 20515163 & 10623 & 14907 & 229 \\
\hline F52 & 20178607 & 10251 & 15410 & 278 \\
\hline F53 & 20471954 & 10453 & 15378 & 263 \\
\hline F54 & 20664862 & 10499 & 15422 & 249 \\
\hline F55 & 20988971 & 10762 & 15428 & 255 \\
\hline F56 & 20721347 & 10503 & 15380 & 257 \\
\hline F57 & 20910003 & 10862 & 15359 & 240 \\
\hline F58 & 20125275 & 10220 & 15431 & 278 \\
\hline F59 & 20209295 & 10464 & 15398 & 252 \\
\hline F60 & 20888234 & 10638 & 15388 & 250 \\
\hline F61 & 20881567 & 10911 & 15376 & 238 \\
\hline F62 & 20774316 & 10505 & 15376 & 254 \\
\hline F63 & 20897163 & 10644 & 15396 & 250 \\
\hline F64 & 20298106 & 10530 & 15414 & 254 \\
\hline F65 & 20914758 & 10682 & 15391 & 266 \\
\hline F66 & 20425513 & 10700 & 15348 & 237 \\
\hline F67 & 20246201 & 10478 & 15400 & 251 \\
\hline F68 & 20724640 & 10727 & 15395 & 222 \\
\hline F69 & 19901820 & 10196 & 15277 & 219 \\
\hline
\end{tabular}

Table 2: The instances 


\begin{tabular}{|c|c|c|c|c|c|c|c|c|}
\hline Name & \multicolumn{2}{|c|}{ Cycle Detection (CD) } & \multicolumn{2}{|c|}{ Local Search (LS) } & \multicolumn{2}{|c|}{$\mathrm{CD}+\mathrm{LS}$} & \multicolumn{2}{|c|}{ Cplex } \\
\hline & coverage $\%$ & sec. & coverage $\%$ & sec. & coverage $\%$ & sec. & UB \% & LB \% \\
\hline$\overline{F 5}$ & $79,8418 \%$ & 85,924 & $95,2727 \%$ & 2033,27 & $95,2986 \%$ & 1415,854 & - & $0,532149 \%$ \\
\hline F6 & $41,6027 \%$ & 94,224 & $40,4891 \%$ & 660,707 & $46,4288 \%$ & 768,83 & $52,3237 \%$ & $47,9199 \%$ \\
\hline F7 & $73,6214 \%$ & 94,911 & $66,0285 \%$ & 1394,22 & $84,3445 \%$ & 982,584 & $96,864 \%$ & $0,254587 \%$ \\
\hline F8 & $57,688 \%$ & 106,236 & $54,8027 \%$ & 1379,88 & $63,34 \%$ & 904,503 & $72,8185 \%$ & $65,9537 \%$ \\
\hline F9 & $80,1467 \%$ & 97,235 & $91,9451 \%$ & 1731,12 & $94,7761 \%$ & 1607,865 & - & $0,0559523 \%$ \\
\hline F10 & $74,7378 \%$ & 88,468 & $62,3175 \%$ & 1280,73 & $87,2839 \%$ & 1069,881 & $98,8187 \%$ & $1,94629 \%$ \\
\hline F11 & $61,3924 \%$ & 126,859 & $66,7614 \%$ & 1047,59 & $74,9865 \%$ & 1404,389 & $82,1221 \%$ & $0,760367 \%$ \\
\hline F21 & $40,1347 \%$ & 121,602 & $36,8315 \%$ & 747,88 & $45,3939 \%$ & 1034,452 & $48,7 \%$ & $46,3055 \%$ \\
\hline F22 & $63,5942 \%$ & 116,423 & $70,2906 \%$ & 1294,07 & $74,834 \%$ & 1026,95 & $78,0683 \%$ & $0,178576 \%$ \\
\hline F23 & $66,9851 \%$ & 114,458 & $71,3575 \%$ & 1429,51 & $79,2129 \%$ & 1217,798 & - & $0 \%$ \\
\hline $\mathrm{F} 24$ & $60,7148 \%$ & 158,824 & $66,9607 \%$ & 896,626 & $70,1393 \%$ & 1297,454 & $73,9213 \%$ & $69,7668 \%$ \\
\hline $\mathrm{F} 25$ & $88,4662 \%$ & 170,242 & $95,5985 \%$ & 1686,94 & $97,7916 \%$ & 1641,512 & $99,6446 \%$ & $6,27393 \%$ \\
\hline F26 & $85,8047 \%$ & 95,253 & $96,741 \%$ & 1099,68 & $98,1487 \%$ & 1881,453 & - & $0,173803 \%$ \\
\hline $\mathrm{F} 27$ & $42,6817 \%$ & 107,546 & $42,8157 \%$ & 539,932 & $48,8265 \%$ & 955,22 & $51,6759 \%$ & $48,229 \%$ \\
\hline F28 & $66,8339 \%$ & 114,504 & $72,4736 \%$ & 1014,69 & $79,6364 \%$ & 1186,474 & $83,9521 \%$ & $74,9001 \%$ \\
\hline F29 & $50,0771 \%$ & 115,549 & $41,1089 \%$ & 730,626 & $54,1612 \%$ & 903,13 & $58,7065 \%$ & $54,4966 \%$ \\
\hline F30 & $84,8831 \%$ & 107,609 & $95,9258 \%$ & 1507,24 & $97,3922 \%$ & 1458,509 & $99,8513 \%$ & $0,0270563 \%$ \\
\hline F31 & $40,0064 \%$ & 124,737 & $37,7534 \%$ & 730,846 & $46,3932 \%$ & 1218,967 & $49,8142 \%$ & $47,329 \%$ \\
\hline F32 & $64,6909 \%$ & 119,247 & $67,8447 \%$ & 1183,59 & $72,4931 \%$ & 1317,357 & $75,5777 \%$ & $0 \%$ \\
\hline F33 & $66,6602 \%$ & 134,036 & $71,0827 \%$ & 1107,8 & $76,4636 \%$ & 1262,726 & $78,7034 \%$ & $2,36324 \%$ \\
\hline F34 & $71,2243 \%$ & 118,108 & $78,0429 \%$ & 1217,94 & $84,7092 \%$ & 1027,416 & $87,6557 \%$ & $0,011673 \%$ \\
\hline F35 & $66,2171 \%$ & 123,646 & $72,5449 \%$ & 1034,61 & $75,6917 \%$ & 1383,226 & $78,5756 \%$ & $0,0888897 \%$ \\
\hline F36 & $83,9962 \%$ & 124,66 & $96,6734 \%$ & 1550,08 & $98,4099 \%$ & 1694,43 & - & $0,625398 \%$ \\
\hline F37 & $87,6432 \%$ & 157,763 & $95,6286 \%$ & 1481,85 & $97,1552 \%$ & 1266,893 & - & $2,59759 \%$ \\
\hline F38 & $78,5477 \%$ & 181,88 & $94,0447 \%$ & 1729,56 & $94,5341 \%$ & 1547,3 & $97,6486 \%$ & $91,3435 \%$ \\
\hline F39 & $49,1384 \%$ & 125,689 & $46,657 \%$ & 769,985 & $54,3828 \%$ & 937,731 & $60,3147 \%$ & $56,9224 \%$ \\
\hline F40 & $84,0879 \%$ & 98,452 & $95,5456 \%$ & 1973,28 & $97,4642 \%$ & 1474,862 & $99,8815 \%$ & $0,118086 \%$ \\
\hline F41 & $63,5636 \%$ & 117,405 & $71,8825 \%$ & 841,027 & $76,1615 \%$ & 1350,755 & $78,712 \%$ & $71,0901 \%$ \\
\hline F42 & $66,4654 \%$ & 108,81 & $70,0535 \%$ & 1374,44 & $74,4103 \%$ & 1146,87 & $77,3379 \%$ & $0,329673 \%$ \\
\hline F43 & $68,7493 \%$ & 109,387 & $70,1778 \%$ & 1049,69 & $80,1439 \%$ & 1235,507 & $84,2964 \%$ & $0 \%$ \\
\hline F44 & $82,3275 \%$ & 93,023 & $95,3856 \%$ & 1263,99 & $97,9221 \%$ & 1636,733 & - & $0,526409 \%$ \\
\hline F45 & $72,5564 \%$ & 102,975 & $73,1345 \%$ & 1058,68 & $84,1291 \%$ & 1225,945 & $88,3394 \%$ & $0 \%$ \\
\hline F46 & $68,4876 \%$ & 111,868 & $70,2297 \%$ & 1224,68 & $80,1255 \%$ & 1051,442 & $84,5057 \%$ & $74,0412 \%$ \\
\hline F47 & $64,9907 \%$ & 135,939 & $70,7747 \%$ & 1280,01 & $74,424 \%$ & 1086,915 & $76,9839 \%$ & $72,7106 \%$ \\
\hline F48 & $81,0323 \%$ & 123,443 & $95,9466 \%$ & 1566,34 & $97,696 \%$ & 1462,433 & $99,8464 \%$ & $0,622013 \%$ \\
\hline F49 & $83,8589 \%$ & 80,558 & $95,4187 \%$ & 2271,06 & $96,745 \%$ & 1689,228 & - & $0,0115108 \%$ \\
\hline F50 & $92,2888 \%$ & 125,596 & $95,6574 \%$ & 1400,9 & $98,1913 \%$ & 1784,846 & - & $0,0909464 \%$ \\
\hline F51 & $40,9997 \%$ & 120,495 & $37,3943 \%$ & 604,313 & $47,0617 \%$ & 898,326 & $49,5654 \%$ & $47,229 \%$ \\
\hline F52 & $88,1002 \%$ & 152,132 & $96,4587 \%$ & 1337,47 & $97,545 \%$ & 1502,032 & - & $1,27357 \%$ \\
\hline F53 & $66,776 \%$ & 117,312 & $70,3306 \%$ & 1119,91 & $79,7436 \%$ & 1068,18 & $84,8421 \%$ & $0 \%$ \\
\hline F54 & $67,4301 \%$ & 113,693 & $68,1214 \%$ & 1158,75 & $73,6662 \%$ & 1099,036 & $77,681 \%$ & $72,2078 \%$ \\
\hline F55 & $65,4833 \%$ & 112,866 & $70,2952 \%$ & 1375,95 & $74,1437 \%$ & 1294,456 & $76,9225 \%$ & $0 \%$ \\
\hline F56 & $67,3372 \%$ & 104,551 & $69,5604 \%$ & 1172,96 & $74,1539 \%$ & 1492,501 & $77,3618 \%$ & $72,2278 \%$ \\
\hline F57 & $49,7666 \%$ & 123,786 & $41,2418 \%$ & 805,927 & $55,558 \%$ & 958,37 & $60,8171 \%$ & $56,3608 \%$ \\
\hline F58 & $86,8067 \%$ & 112,242 & $97,1489 \%$ & 1529,83 & $98,042 \%$ & 1684,702 & $99,5915 \%$ & $4,44063 \%$ \\
\hline F59 & $67,9255 \%$ & 122,507 & $72,0547 \%$ & 1193,74 & $76,2406 \%$ & 1021,365 & $79,0792 \%$ & $0,686095 \%$ \\
\hline F60 & $65,2924 \%$ & 112,944 & $71,7 \%$ & 1106,82 & $74,3476 \%$ & 887,437 & $76,7249 \%$ & $0,0262636 \%$ \\
\hline F61 & $53,8853 \%$ & 130,089 & $42,5093 \%$ & 947,872 & $59,0629 \%$ & 799,86 & $63,6467 \%$ & $59,0989 \%$ \\
\hline F62 & $66,2672 \%$ & 104,52 & $71,5899 \%$ & 1574,53 & $74,083 \%$ & 1295,96 & $77,0095 \%$ & $72,224 \%$ \\
\hline F63 & $65,7345 \%$ & 111,337 & $71,5625 \%$ & 1152,86 & $74,3454 \%$ & 1465,167 & - & $0 \%$ \\
\hline F64 & $68,0427 \%$ & 163,426 & $68,713 \%$ & 1244,44 & $73,2866 \%$ & 1254,286 & $75,7337 \%$ & $68,9843 \%$ \\
\hline F65 & $66,7843 \%$ & 136,359 & $72,3228 \%$ & 1402,47 & $74,902 \%$ & 1384,469 & $77,4342 \%$ & $73,616 \%$ \\
\hline F66 & $42,7375 \%$ & 143,583 & $44,0755 \%$ & 916,562 & $49,4907 \%$ & 1274,303 & $51,9139 \%$ & $49,7468 \%$ \\
\hline F67 & $59,806 \%$ & 118,981 & $69,9529 \%$ & 1540,5 & $75,5733 \%$ & 1023,267 & $78,388 \%$ & $71,77 \%$ \\
\hline F68 & $50,1931 \%$ & 116,625 & $43,5531 \%$ & 1026,86 & $55,9932 \%$ & 891,151 & $61,0669 \%$ & $57,0673 \%$ \\
\hline F69 & $85,6529 \%$ & 67,345 & $97,6251 \%$ & 1451,49 & $98,0405 \%$ & 1043,062 & $99,8893 \%$ & $93,3489 \%$ \\
\hline
\end{tabular}

Table 3: Computational Results 


\section{References}

[1] Ahuja, K.R., Ergun, Ë., Orlin, J.B., Punnen, A.P., A survey of very large-scale neighborhood search techniques, Discrete Applied Mathematics, 123, 1-3 (2002), 75-102.

[2] R. Ahuja, T.L. Magnanti, J. Orlin, Network Flows Prentice Hall, 1993.

[3] E. Amaldi, A. Capone, and F. Malucelli. Discrete models and algorithms for the capacitated location problem arising in UMTS network planning. In Proceedings of the 5th International Workshop on Discrete Algorithms and Methods for Mobile Computing and Communications (DIAL-M), pages 1-8. ACM, 2001

[4] E. Amaldi, A. Capone, F. Malucelli and C. Mannino. Optimization problems and models for planning cellular networks, to appear in Handbook of Optimization in Telecommunication, Eds. M. Resende and P. Pardalos, Springer Science.

[5] C.A. Balanis, Antenna Theory: Analysis and Design, John Wiley \& Sons, New York, 1982

[6] R. Beutler, Frequency Assignment and Network Planning for Digital Terrestrial Broadcasting Systems, Kluwer Academic Pubishers, 2004

[7] ILOG-CPLEX solver, http://www.ilog.fr

[8] A. Eisenblätter, H. Geerdes, T. Koch, A. Martin, R. Wessly. UMTS Radio Network Evaluation and Optimization Beyond Snapshots. Technical Report ZR-04-15, ZIB, 2004.

[9] J. Kalvenes, J. Kennington and E. Olinick, "Base Station Location and Service Assignments in W-CDMA Networks," INFORMS Journal on Computing (forthcoming).

[10] C. Mannino, F. Rossi, S. Smriglio, The Network Packing Problem in Terrestrial Broadcasting, to appear in Operations Research

[11] R. Mathar, M. Schmeinck, Optimisation Models for GSM Radio, International Journal of Mobile Network Design and Innovation, 1, 1, 2005, 70-75.

[12] D. Parsons, The Mobile Radio Propagation Channel, John Wiley \& Sons, 1998

[13] Regional Radiocommunication Conference (RRC-06), Final Act, http://www.itu.int/ITU-R/conferences/rrc/rrc-06/index.asp.

[14] F. Rossi, A. Sassano, S. Smriglio, Models and Algorithms for Terrestrial Digital Broadcasting, Annals of Operations Research, no. 107, pp. 267-283 2001 